International Journal of Remote Sensing

July 2007; 28 (17) 3953-3962

http://dx.doi.org/10.1080/01431160601047789

(C) 2007 Taylor \& Francis Ltd
Archimer http://www.ifremer.fr/docelec/ Archive Institutionnelle de l'Ifremer

The original publication is available at http://www.tandf.co.uk/journals/

\title{
Detection of wave fronts in the Indian Ocean from geostationary sunglint satellite imagery
}

\author{
A. Turiel ${ }^{a,}{ }^{*} ;$ J. Isern-Fontanet ${ }^{\text {b. }}$; E. Garcia-Ladona ${ }^{\text {a. }}$ J. A. Young ${ }^{c}$ \\ a Institut de Ciències del Mar - CSIC. Passeig Marítim de la Barceloneta, 37-49. 08003 Barcelona, Spain \\ b Laboratoire de Physique des Océans. Ifremer. BP 70, F-29280 Plouzane, France and Laboratoire \\ d'Océanographie Spatiale. Ifremer, BP 70, F-29280 Plouzane, France \\ c University of Winsconsin-Madison, WI 53706 \\ *: Corresponding author : turiel@icm.csic.es
}

\begin{abstract}
:
We demonstrate the potential of monitoring coherent wave fronts on the tropical ocean surface using an advanced detection algorithm applied to visible sunglint-affected imagery from geostationary satellites. Data from MeteoSat are processed with the advanced analysis technique to reveal transient wave activity near and to the west of the Mascarene Ridge of the western Indian Ocean. The technique is based on a wavelet-based singularity analysis which enhances the subtle but highly coherent wave patterns in the meteorological satellite data. It is likely that the patterns represent internal ocean waves, a pathway to ocean mixing and climate. The prospect for using this detection algorithm in greatly expanded satellite global surveys of wave activity is outlined.
\end{abstract}




\title{
Detection of wave fronts in the Indian Ocean from geostationary sunglint satellite imagery
}

\author{
Antonio Turiel†, Jordi Isern-Fontanet $\ddagger$ Emilio Garcia-Ladona $\dagger$, and John A. Young§ \\ † Institut de Ciències del Mar - CSIC. Passeig Marítim de la Barceloneta, 37-49. 08003 Barcelona. Spain \\ ‡ Laboratoire de Physique des Océans. Ifremer. BP 70, F-29280 Plouzane, France \\ and \\ Laboratoire d'Ocanographie Spatiale. Ifremer. BP 70, F-29280 Plouzane, France \\ $\S$ University of Winsconsin-Madison, WI 53706. USA \\ (Received 00 Month 200x; In final form 00 Month 200x)
}

\begin{abstract}
We demonstrate the potential of monitoring coherent wave fronts on the tropical ocean surface using an advanced detection algorithm applied to visible sunglint-affected imagery from geostationary satellites. Data from MeteoSat are processed with the advanced analysis technique to reveal transient wave activity near and to the west of the Mascarene Ridge of the western Indian Ocean. The technique is based on a wavelet-based singularity analysis which enhances the subtle but highly coherent wave patterns in the meteorological satellite data. It is likely that the patterns represent internal ocean waves, a pathway to ocean mixing and climate. The prospect for using this detection algorithm in greatly expanded satellite global surveys of wave activity is outlined.
\end{abstract}

\section{Introduction}

It is increasingly evident that gravity-induced Internal Waves (IW) in the ocean are a direct link to mixing processes (Rudnick et al., 2003; Navrotsky et al., 2004) that affect ocean dynamics, biological productivity, and global climate (Munk and Wunch, 1998; Wunsch and Ferrari, 2004; Garret, 2003). These waves are concentrated in large (hundreds of $\mathrm{km}$ ) patches centered near strong underwater topographical features, their generation being mostly a result of semidiurnal tidal currents (Ray and Mitchum, 1996). Global maps of energetic patches have been estimated from moored buoy data (Morozov, 1995) and satellite altimetry (Kantha and Tierney, 1999). An atlas (Jackson, 2004) of concentrated soliton events from aircraft and satellite radar demonstrates the ubiquity of the waves at the surface.

The geographical pathway from global tides to small-scale mixing is a challenging concoction of wave dispersion, interacting scales, and decay (Rudnick et al., 2003). In situ measurements combined with theory or numerical modeling have created plausible routes for the energy (e.g. Alford, 2003; Morozov, 1995; Lozovatsky et al., 2003). The dominant tidal IW is thought to redistribute energy horizontally at a speed of up to $2.5 \mathrm{~m} / \mathrm{s}$, with an initial wavelength of about $120 \mathrm{~km}$, maintaining high energy level over a distance of about three wavelengths $(400 \mathrm{~km})$. Energy loss is likely due to the creation and breaking of solitary wave fronts and energy cascade to smaller wave scales with ultimate turbulent dissipation in velocity shear zones.

Ideally, quasi-continuous "synoptic" and high-resolution surveys are needed to define the space-time variability of these transient elements at key locations. This may be optimally achieved by expanding the capabilities of satellite remote sensing and signal detection. The work reported here is intended to be one such step toward enhanced monitoring of the sea surface.

Corresponding author. Email: turiel@icm.csic.es

International Journal of Remote Sensing

ISSN 0143-1161 print/ ISSN 1366-5901 online (C)2005 Taylor \& Francis Ltd

http://www.tandf.co.uk/journals

DOI: $10.1080 / 01431160 \mathrm{xxxxxxxxxxxxx}$ 
IWs cause vertical displacements of water parcels which are at minimum (centimeters) at the sea surface, comparable to the accuracy of individual satellite altimetric measurements. These measurements have allowed to identify the existence of IWs (Ray and Mitchum, 1996), but noise and space-time sampling limitations preclude mapping of an instantaneous two-dimensional IW field from altimetry. However, bandpass filtering (Kantha and Tierney, 1999; Egbert and Ray, 2000) of altimetry shows that IW variability at tidal periods is energetically significant and is associated with ocean bottom topography.

Instantaneous satellite observations of IWs are indirect, based on organized patterns of enhanced smallscale (cm-10m wavelength) surface waves. The roughening has been thought to result from intense flow convergence in the IW current field, but it is possible that the strongest IW currents themselves may generate localized roughening at the larger scales of wave spacing as observed here. Visual evidence of roughening was noted by ancient mariners and, in modern times by astronauts (Jackson, 2004) and oceanographers (Osborne and Burch, 1980). The first satellite images of IWs were by synthetic aperture radar (SAR) measurements (Apel et al., 1975), which identified apparent IWs in the surface return at wavelengths corresponding to capillary waves. Recently, samples of high-resolution MODIS imagery (Jackson, 2004) have shown coherent patterns in visible imagery.

Polar-orbiting satellites are limited by the relatively small size of the image "footprint" and the infrequent sampling of a particular location; in fact, SAR-detected wave-like patterns can only be associated with highfrequency, short-wavelength IWs. To observe low-frequency, long-wavelength IWs, well-focused, dedicated experiments, with simultaneous in-situ and remote sensing observations are required (Kim et al., 2005). On the contrary, geostationary satellites such as MeteoSat series are capable of observations better suited to surveying specific large regions for a long period of time. Limitations of geostationary imagery are relatively low spatial resolution (2.5 km for MeteoSat) and "noise" contributed by small (sub-pixel) cumulus clouds. However, with the aid of geostationary satellites sequential time variations (of order of minutes) may be seen, and arbitrarily long periods may be sampled at the same location. These capabilities have the potential for identifying propagation and decay of individual coherent waves in the ocean under conditions where large-scale, persistent clouds are absent, and some sunglint is present. Waves can be detected due to their space-time coherency and after enhancement by an advanced signal-processing technique.After enhancement by an advanced signal-processing technique, waves can be detected due to their space-time coherency.

\section{Validity and limitations of the data used in this study}

This study is based on visible imagery for the tropics from the meteorological geostationary satellite MeteoSat 5, which is stationed at longitude $63 \mathrm{E}$ on the equator. We have used full-range digital images from the visible channel of MeteoSat-5 to detect IWs and thermal infrared images from the same satellite to validate the oceanic origin of the detected structures. The time step between images is 30 minutes. The visible wavelengths (range 0.5 to 0.9 microns) have a spatial resolution of $2.5 \mathrm{~km}$ at the sub-satellite point while thermal infrared data (range 10.5 to 12.5 microns) have a spatial resolution of $5 \mathrm{~km}$ at the sub-satellite point. The analyzed visible images have a size of $500 \times 500$ pixels. All the data used were provided by EUMETSAT.

MeteoSat 5 luminosity data is linearly coded using a single byte, which limits the range of subtle variations which can be detected. In this demonstration, results were obtained applying our technique to the higher-contrast "sunglint" areas of scattering from the ocean surface. This illumination increases the contrast in the image, so that subtle differences between neighboring pixels may represent variations which actually occur on the sub-pixel scale; the sunglint effect has been used, for instance, to highlight internal tide effects (Pingree and New, 1995). The sunglint pattern passes by the sub-satellite longitude at local noon (around 8:00 GMT). Usable sun glint illuminated the domain center for about $2 \mathrm{~h}$; the center of the glint was within the domain for about $1.5 \mathrm{~h}$, twice that of the subsolar point. The pattern has a variable width, depending on sea surface roughness. A width of 15 deg longitude may illuminate a fixed location for about two hours. This allows a time sequence of consecutive images (every 30 minutes) for each day to be compared for tropical regions near the satellite most months of the year. Some subtropical locations may be sampled in the local summer season. 
We have analyzed a total of 12 days (about 100 images) distributed into four periods: July 5-7 2004, September 25-27 2004, December 25-27 2004 and March 10-12 2005. July and September images were unusable due to the weak sun glint enhancement relative to the presence of clouds. In March, only one day was sufficiently enough cloud-free to allow observation of the area.

To estimate the temperature of the patterns identified in the visible images we have bilinearly interpolated the thermal infrared data onto the finer grid of visible images.

\section{Singularity analysis for enhanced pattern extraction}

A crucial feature of our detection algorithm is that for each image in a sequence the inter-pixel differences are enhanced and so spatially coherent signatures (such as long wave fronts) can be identified. Singularity analysis is based on proposing a generalized Taylor expansion for studying the local behaviour of a function around a given point. For an image $I(\vec{x})$, we will say that it permits a pseudo-Taylor expansion (Arneodo et al., 1995) of index $H_{0}$ around a point $\vec{x}_{0}$ if the following expression holds:

$$
\left|I(\vec{x})-I\left(\vec{x}_{0}\right)\right|=A_{0}\left|\vec{x}-\vec{x}_{0}\right|^{H_{0}}+O\left(\left|\vec{x}-\vec{x}_{0}\right|^{H_{0}}\right)
$$

The exponent $H_{0}$ is known as the local Hölder exponent of the function $I$ at the point $\vec{x}_{0}$, and measures the degree of regularity (if positive) or irregularity (if negative) of the function around that point. Sharp transitions in an image are associated with minimum values of $H_{0}$. Local singularity exponents allow us to detect and to characterize transitions locally, taking into account their sharpness only and disregarding the associated amplitude $A_{0}$. For that reason, local singularity analysis provides a finer detection than global thresholding methods (Turiel and Parga, 2000).

In practice, some processing issues (as for instance the necessity of using adequate interpolation schemes for discretized data (Arneodo et al., 1995)) force the introduction of wavelet-based techniques for performing singularity analysis (Mallat, 1999). In addition, analyzing the gradient of the intensity, $\nabla I$, provides a finer localization of the structures of interest (Turiel and Parga, 2000). Hence, for each image $I(\vec{x})$ we take the modulus of its gradient, $|\nabla I|(\vec{x})$, at each point $\vec{x}$. Then, given a wavelet $\Psi(\vec{x})$ (Mallat, 1999), we define the wavelet transform of the gradient at the point $\vec{x}$ and the scale $r$ as:

$$
T_{\Psi}|\nabla I|(\vec{x}, r) \equiv \int d \vec{y}|\nabla I|(\vec{y}) \frac{1}{r^{2}} \Psi\left(\frac{\vec{x}-\vec{y}}{r}\right)
$$

Now we will say that the image has a local singularity exponent $h(\vec{x})$ at a given resolution $r$ for the point $\vec{x}$ if the following relation holds:

$$
T_{\Psi}|\nabla I|(\vec{x}, r)=\alpha(\vec{x}) r^{h(\vec{x})}+O\left(r^{h(\vec{x})}\right)
$$

where $\alpha(\vec{x})$ is a multiplier, independent of the resolution $r$. In this paper, the singularity exponents are experimentally determined by applying the formula above to the images for a wavelet $\Psi=\left(1+|\vec{x}|^{2}\right)^{-2}$ at different resolutions $r$ ranging from 1 to 16 pixels (namely,from 2.5 to $40 \mathrm{~km}$ ). We obtain the singularity exponent $h(\vec{x})$ at each point as the slope of the linear regression of $\log T_{\Psi}|\nabla I|(\vec{x}, r)$ versus $\log r$. The values of $h(\vec{x})$ typically fall in the range $(-1,2)$; negative values correspond to sharp transitions, and are typically associated to edges and contours (Turiel and Parga, 2000). We define the set comprising edges and other sharp transition as the Most Singular Manifold, MSM (Turiel et al., 1998; Turiel and Parga, 2000; Turiel et al., 2005). In this work, we defined the MSM as the $45 \%$ most singular points. This truncation is intended to reduce small-scale noise or contamination by scattered groups of small cumulus clouds (Roux et al., 2000). Boundaries of larger cloud groups may be visible in the MSM, but do not have the smooth geometry or slow propagation speeds of coherent waves on the ocean. 


\section{Results}

We demonstrate our technique for the region surrounding the Mascarene Ridge system of the western Indian Ocean, where in situ (Konyaev et al., 1995; Lozovatsky et al., 2003) and space (Kantha and Tierney, 1999) observations have shown internal ocean waves to be common. The complex is centered near $60.5 \mathrm{E}, 11.5 \mathrm{~S}$ and is located near the MeteoSat sunglint maximum in SH summer, and 11.5 degrees to its south at the equinoxes.

Fig. 2 shows the application of the singularity analysis technique to satellite images. The December 26, 2004 date is one of nearly optimum sun glint for this location. The clearer relatively cloud-free regions show MSM patterns with coherent structures which are interpreted as ocean wave signatures (arcs traced from the MSM field). These signatures are considered robust, as their lengths of up to $200 \mathrm{~km}$ are defined by several hundred pixels. The MSM fields accentuate these signatures, which are nearly obscure in the unprocessed images.

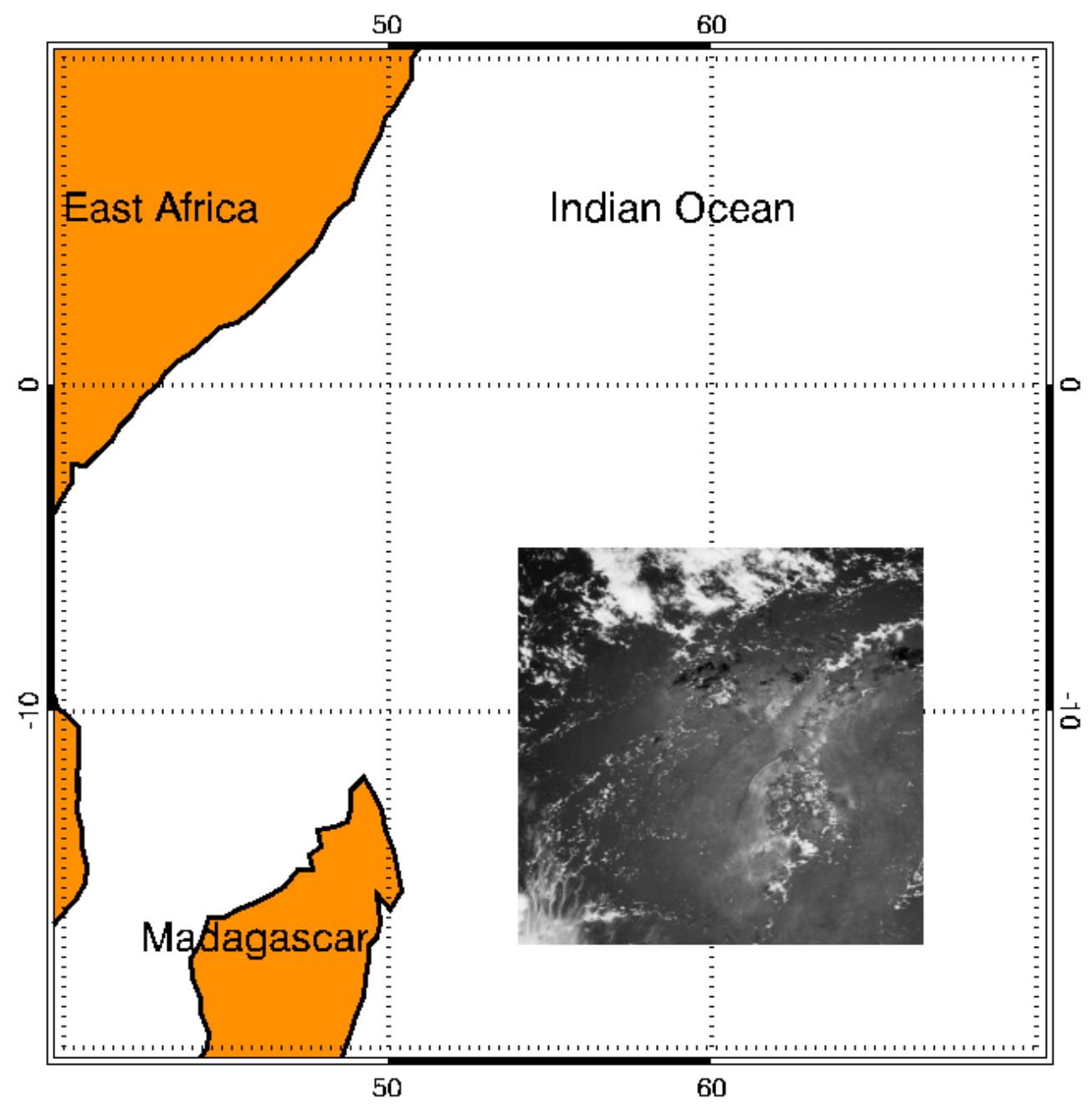

Figure 1. Visible channel image from Meteosat 5, acquired at 8:00 GMT on December 26th, 2004. The intensities have been represented in a logarithmic gray scale to enhance details. One wave front is visually apparent near $62^{\circ} \mathrm{E}, 12^{\circ} \mathrm{S}$.

We used infrared images to verify that the frontal features on the images originate at the ocean surface, and are not due to atmospheric features such as clouds. The infrared sensor on the MeteoSat 5 has been used to create a blackbody emission temperature. Warm temperatures correspond to the sea surface with clear skies, while cool temperatures are due to clouds above the surface. The fronts are seen to be warm 

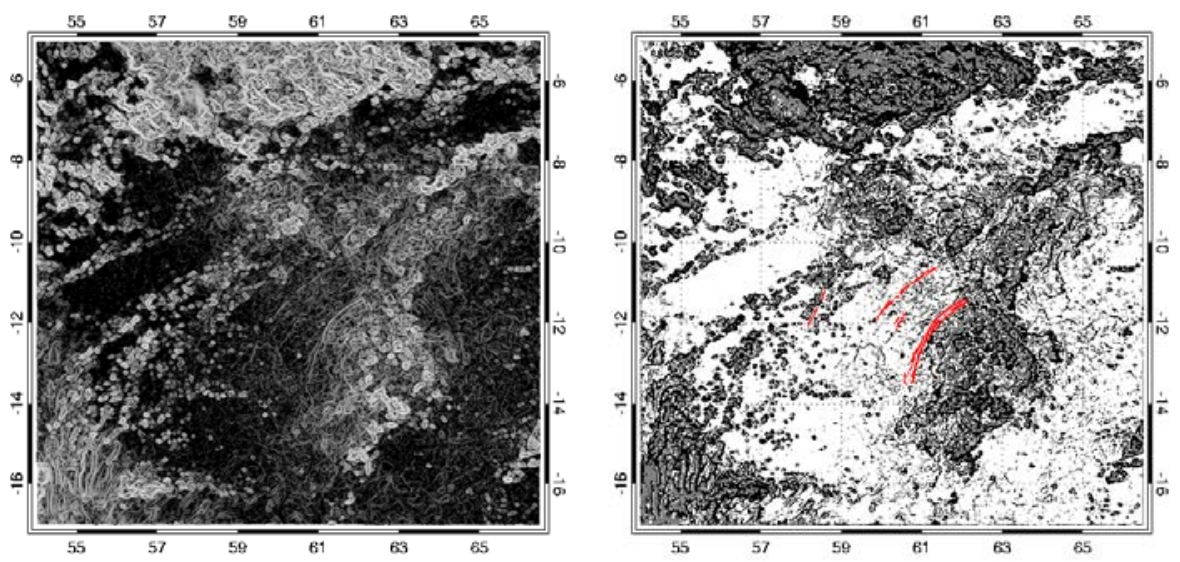

Figure 2. Illustration of MSM detection method applied to the image in Figure 1. Left: Graylevel representation of the singularity exponents obtained from the image above. The smallest values (most singular points) are depicted using bright colors, while less singular ones are darker. Right: Most Singular Manifolds (MSM), as extracted from the image on the left. Cloud systems have a rich, singular structure (Roux et al., 2000) and contain many MSM points; MSM over open areas are sparser. Red arcs are wave fronts drawn by hand from MSM features. More fronts are seen in MSM fields than are identifiable in the unprocessed image.

(above $295 \mathrm{~K}$ ), which therefore makes their ocean surface origin plausible.

Fig. 3 shows a time sequence of wave fronts on the preceding day and their proximity to the Mascarene Ridge topography. The nonlinear color scale emphasizes the higher peaks of the ridge, with an elevated channel between them. Interestingly, the most distinct wave fronts are found to the west of the ridge, and shorter, less distinct ones are found to the east. Figs. 3 and 4 display the fronts detected for the three days Dec. 25-27; a similar preference for waves to the west is seen. The repetitive pattern of fronts during all these days provides further evidence on the oceanic origin of the fronts. These waves seem to be propagating westward, with an approximate speed of about 1 pixel/image, or $5 \mathrm{~km} / \mathrm{h}(1.6 \mathrm{~m} / \mathrm{s})$. These speeds are consistent with deep internal gravity wave propagation in the ocean (e.g. Lozovatsky et al., 2003). The speeds and westward propagation are consistent with internal wave energy radiating away from the ridge toward the west; in situ observations (Lozovatsky et al., 2003; Jackson, 2004; Apel et al., 1985) have indicated wave energy radiating away from the ridge on the east side; there is no previous report on waves propagating to the west. Both propagations are consistent with the notion of tidal currents generating IWs at the ridge, and with observed wave fluxes in the Pacific Ocean near the Hawaiian ridge (Rudnick et al., 2003). The similarity of some wave patterns (e.g., the eastern side of the channel) on successive days could be due to the approximate time synchronization of the satellite observations with the semidiurnal tides during these three days. One wave front is seen at a distance $300 \mathrm{~km}$ from the ridge, which corresponds to a three-day travel time at the speeds estimated above.

We believe that these waves are unrelated to the Indonesian tsunami of 26 December 2004, as they are visible the preceding and successive day and in other months (one of the March images). The tsunami apparently generated internal waves of short wavelength viewed by the MODIS satellite over the Sri Lankan coastal shelf (Santek and Winguth, 2006). The latter image has been obtained for the equinoctial date of 11 March, 2005, when sun glint at the ridge location is not optimum. Despite this, all four MSM images appear to show numerous wave fronts. Clouds obscured the imagery on neighboring days. No waves were identified for the equinoctial dates of 25-27 September 2004, probably as a result of interference by cumulus cloud fields; as expected, none were identified at this latitude for the winter dates 5-7 June 2004.

Fig. 4 summarizes the primary wave fronts in this study, superimposed on the Mascarene Ridge bathymetry. The prevalence of waves near the ridge, its gap, and to the west suggest complex generation processes that may involve radial spreading from the gap and amplification over the shallow northern plateau. 

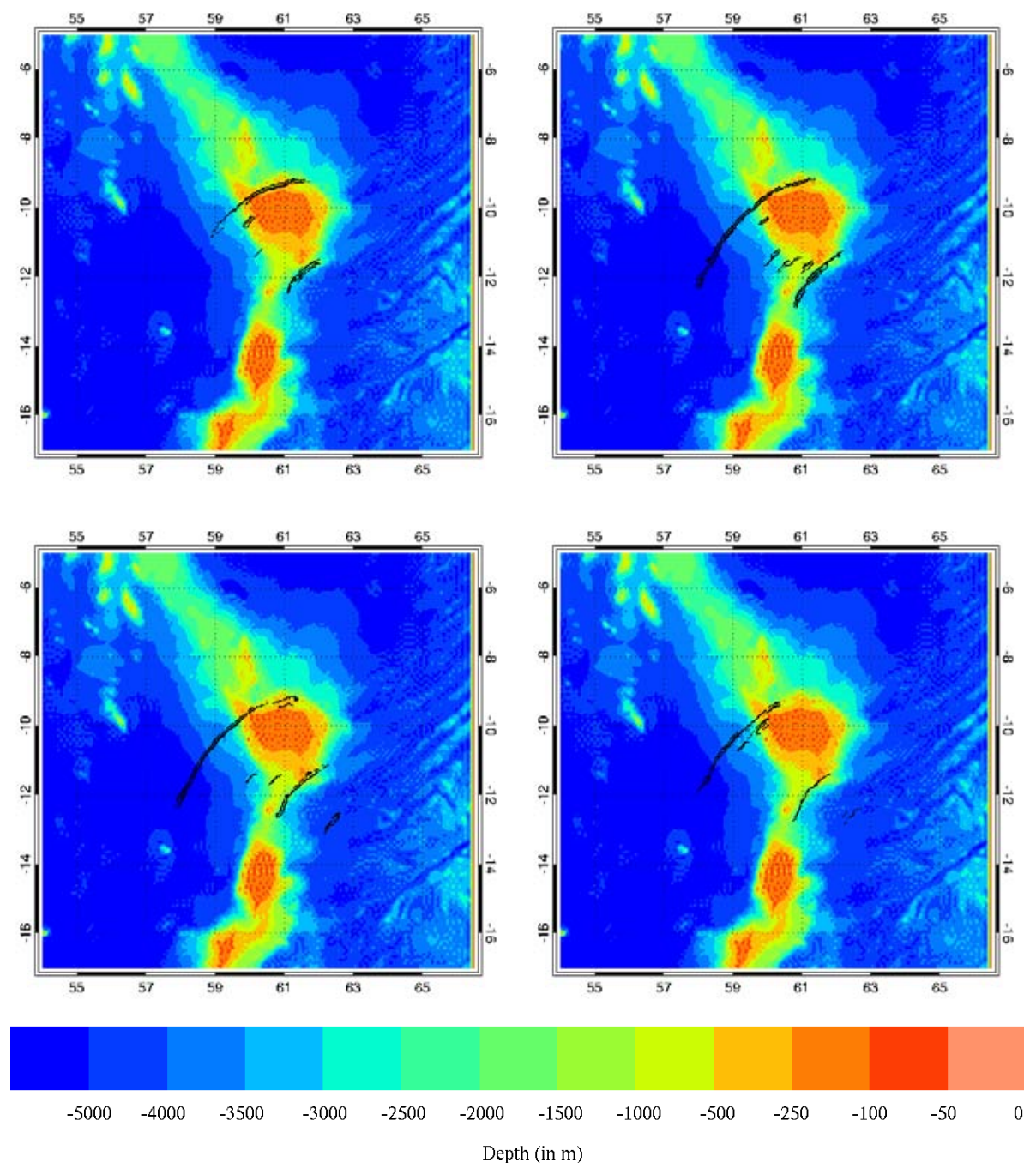

Figure 3. Assessed MSM wave fronts (in black) overlaid on Mascarene Ridge bathymetry. Data is for December 25th, 2004, at 7:30 (top, left), at 8:00 (top, right), at 8:50 (bottom, left) and at 9:00 (bottom, right; all times are GMT)

\section{Discussion}

This Indian Ocean MeteoSat study could be expanded to study wave occurrences at other locations in that ocean, and in other seasons. This is because the satellite's view of sunglint illumination extends over all longitudes of the Indian Ocean. The glint's image radius is about $7.5 \mathrm{deg}$ longitude, and at least $5 \mathrm{deg}$ latitude. It is centered at a latitude equal to about half the sub-solar latitude. Thus, there is a seasonal cycle of optimum possible sun glint illumination, its latitude varying between the equator at the equinoxes to 11.7 degrees at the solstices. Conservatively, partial illumination may extend as far as about 17 degrees latitude into the summer hemisphere at solstice. On an annual basis, barring cloud cover, the potential duration of sun glint monitoring is more than four months within about 12 degrees of the equator, with a maximum of 12 months near the equator.

Finally, our results suggest some steps for expanding satellite research and surveillance of wave signatures using advanced image processing techniques. (i) Historical data sets from other geostationary satellites could be reanalyzed with the procedure used here. This would greatly expand the number of recorded events, extend the survey to other oceans and seasons, and form the basis for a global atlas for the tropical belt. (ii) The design of future satellite data systems should aim to expand the luminosity information content to allow sensitive detection, as that provided by MSM algorithm, in regions of lower contrast. This would allow detection over a larger area outside the sunglint zone, with capabilities exceeding the human 

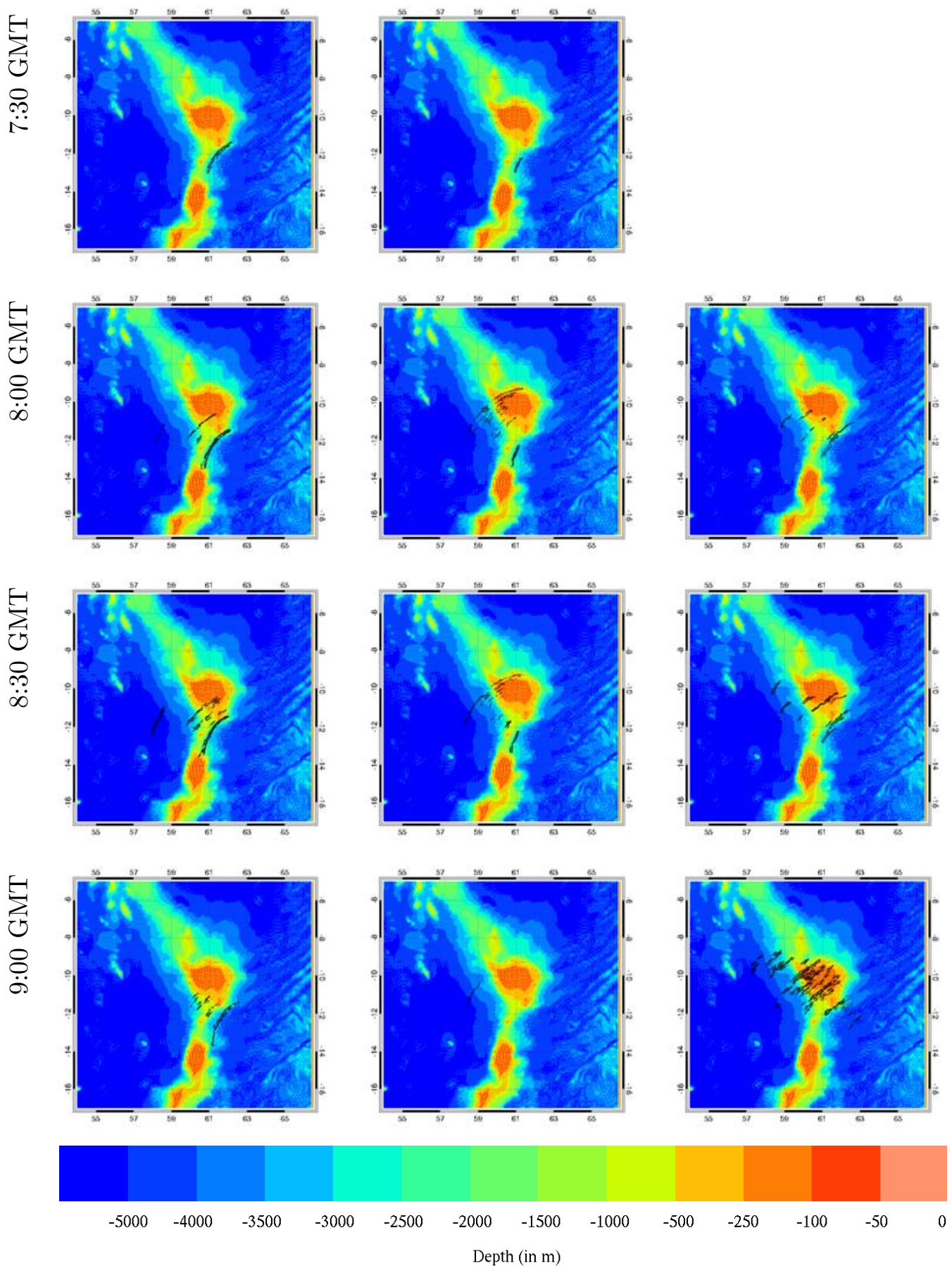

Figure 4. Collection of all MSM wave fronts (in black) assessed in this study, overlaid on Mascarene Ridge bathymetry.

eye limit of 30 gray scales (Russ, 1999). (iii) These technique improvements could be introduced into polar orbiting satellites (e.g., MODIS), which could widen coverage with a global "constellation" of sensors.

\section{Acknowledgements}

This is a contribution to IMAGEN (Spanish R+D Plan: REN2001-0802-C02-02) and MERSEA projects (EU AIP3-CT-2003-502885). A. Turiel is funded by a Ramon y Cajal contract from the Spanish Ministry of Education. J. Isern-Fontanet is funded by a post-doctoral grant by the Spanish Ministry of Education. J. Young's visit to ICM was provided by a sabbatical and Graduate School WARF funding from the 
University of Wisconsin-Madison. We thank EUMETSAT for leaving us their data. We thank Prof. Iossif Lossovatzki and Dr. Pere Puig by their helpful comments during the development of this study.

\section{References}

Alford, M., May 2003. Redistribution of energy available for ocean mixing by long-range propagation of internal waves. Nature 423, 159-162.

Apel, J., Byrne, H., Proni, J., Charnell, R., 1975. Observations of oceanic internal and surface waves from the earth resources technology satellite. J. Geophys. Res. 80 (6), 865-881.

Apel, J., Holbrook, J., A.K.Liu, Tsai, J., 1985. The Sulu Sea Internal Soliton Experiment. Journal of Physical Oceanography 15 (12), 1625-1651.

Arneodo, A., Argoul, F., Bacry, E., Elezgaray, J., Muzy, J. F., 1995. Ondelettes, multifractales et turbulence. Diderot Editeur, Paris, France.

Egbert, G., Ray, R., June 2000. Significant dissipation of tidal energy in the deep ocean inferred from satellite altimeter data. Nature 405, 775-778.

Garret, C., September 2003. Internal tides and ocean mixing. Science 301, 1858-1859.

Jackson, C. R., February 2004. An Atlas of Internal Solitary-like Waves and their Properties, 2nd Edition. Global Ocean Associates, Alexandria, USA.

Kantha, L., Tierney, C., 1999. Global baroclinic tides. Progress in Oceanography 40, 163.

Kim, D., Nam, S., Kim, H., Moon, W., Kim, K., 2005. Can near-inertial internal waves in the East Sea be observed by synthetic aperture radar? Geophysical Research Letters 32, L02606, doi:10.1029/2004GL021532.

Konyaev, K., Sabinin, K., Serebryany, A., 1995. Large-amplitude internal waves at the mascarene rigde in the indian ocean. Deep Sea Research I 42 (11-12), 2075-2091.

Lozovatsky, I., Morozov, E., Fernando, H., 2003. Spatial decay of energy density of tidal internal waves. Journal of Geophysical Research 108 (C6), 3201, doi:10.1029/2001JC001169.

Mallat, S., 1999. A Wavelet Tour of Signal Processing. Academic Press, 2nd Edition.

Morozov, G., 1995. Semidiurnal internal wave global field. Deep-Sea Research 42, 135.

Munk, W., Wunch, K., 1998. Abyssal recipes ii: energetics of tidal and wind mixing. Deep-Sea Research 45, 1977.

Navrotsky, V., Lozovatsky, I., Pavlova, E., Fernando, H., 2004. Observations of internal waves and thermocline splitting near a shelf break of the Sea of Japan (East Sea). Continental Shelf Research 24, $1375-1395$.

Osborne, A., Burch, T., 1980. Internal solitons in the andaman sea. Science 208, 451.

Pingree, R., New, A., 1995. Structure, seasonal development and sunglint spatial coherence of the internal tide of the Celtic and Armorican shevels in the Bay of Biscay. Deep-Sea Research 42 (2), 245-284.

Ray, R., Mitchum, G., 1996. Surface manifestation of internal tides generated near Hawaii. Geophysical Research Letters 23, 2101.

Roux, S. G., Arneodo, A., Decoster, N., 2000. A wavelet-based method for multifractal image analysis. iii. applications to high-resolution satellite images of cloud structure. Eur. Phys. J. B 15, 765-786.

Rudnick, D., Boyd, T., Brainard, R., Carter, G. S., Egbert, G., Gregg, M., Holloway, P., Klymak, J., Kunze, E., Lee, C., Levine, M., Luther, D., Martin, J., Merrifield, M., Moum, J., Nash, J., Pinkel, R., Rainville, L., Sandford, T., July 2003. From tides to mixing along Hawaiian Ridge. Science 301, 355-357.

Russ, J., 1999. The image processing handbook. Bocaraton SL:CRG.

Santek, D., Winguth, A., 2006. A satellite view of internal waves induced by the indian ocean tsunami. International Journal of Remote Sensing (In press).

Turiel, A., Isern-Fontanet, J., García-Ladona, E., Font, J., September 2005. Multifractal method for the instantaneous evaluation of the stream function in geophysical flows. Physical Review Letters 95 (10), 104502, doi:10.1103/PhysRevLett.95.104502.

Turiel, A., Mato, G., Parga, N., Nadal, J. P., 1998. The self-similarity properties of natural images resemble those of turbulent flows. Physical Review Letters 80, 1098-1101. 
Turiel, A., Parga, N., 2000. The multi-fractal structure of contrast changes in natural images: from sharp edges to textures. Neural Computation 12, 763-793.

Wunsch, C., Ferrari, R., 2004. Vertical mixing, energy, and the general circulation of the oceans. Annu. Rev. Fluid Mech. 36, 281-314. 